Website: http://revistas.lamolina.edu.pe/index.php/acu/index

(C) Universidad Nacional Agraria La Molina, Lima - Perú

\title{
Modelación de la curva de lactación de vacas gir y cruces gir por holstein (F-1) en el trópico peruano
}

\author{
Modeling of lactation curve of gir and gir-holstein (F-1) cows in the peruvian tropic \\ Pedro Huamán Quispe'; José Almeyda Matias²; Julio Isique Huaroma ${ }^{1}$ \\ * Autor de correspondencia
}

\section{Resumen}

El presente estudio se realizó en la región San Martín con el objetivo de estudiar tres métodos de regresión: lineal simple, cuadrática y no lineal de Wood; para modelar y analizar la curva de lactación de vacas cruzadas F-1 y Gir lechero de primera lactación bajo condiciones de trópico. Para tal efecto se utilizó los registros de producción de leche de 60 vacas, de las cuales 30 correspondieron a cruzadas F-1 y 30 a Gir lechero. Las vacas cruzadas F-1 estuvieron estabuladas donde recibieron una ración alimenticia compuesta por forraje de corte con suplemento concentrado y ordeñadas dos veces/día y las Gir lechero fueron criadas extensivamente alimentadas al pastoreo con suplemento de concentrado y ordeñadas una vez/día, sin presencia de la cría. Los resultados demuestran que el método no lineal de Wood fue el más confiable para explicar e interpretar el modelamiento y las características de la curva de lactación de ambos grupos de vacas con coeficientes de determinación de: 0,9312 y 0,9108 respectivamente. Asimismo al desarrollar las ecuaciones de la metodología se determinó los valores de las siguientes características de la curva de lactación: 4 031,15 y 1 031,10 kg de leche por campaña; 85 y 55 días de duración del periodo parto-pico de producción y 17,22 y 4,28 kg de leche/día como nivel de producción en el pico para las vacas cruzadas F-1 y Gir lechero respectivamente; además se estimó una persistencia de 8,4 por ciento después del pico de producción para ambos grupos.

Palabras clave: Curva de lactación; vacas cruzadas F-1 y Gir; métodos, modelamiento y análisis.

\begin{abstract}
This study was carried out in the San Martin Region of Peru aiming to model and analyze the lactation curve of F1 crossbred cows and Gir first-lactation dairy cows under tropic conditions. Three regression methods were used: simple regression, quadratic and Wood nonlinear. The data used in this study contained milk production records from 60 cows; 30 from F1 crossbred cows and 30 from Gir first-lactation dairy cows. F1 crossbred cows were stabled, fed with a forage-based ration with concentrated supplements and milked twice a day whereas Gir first-lactation dairy cows were extensively bred, pasture-fed with concentrated supplements and milked once a day without the presence of the calf. Results demonstrated Wood's model was the most reliable method to explain and interpret the modeling and traits of lactation curves for both groups of cows (Coefficient of determination: 0.9312 and 0.9108 , respectively). At the same time, while solving methodology equations, values from the following traits of the curve of lactation were determined: 4031.15 and $1031.10 \mathrm{~kg}$ of milk per season; 85 and 55 days of peak-calving season period production and; 17,22 and 4,28 $\mathrm{kg}$ of milk/day as the production peak level for F1 crossbred and Gir first-lactation dairy cows, respectively. Moreover, a persistency of $8,4 \%$ after production peak was estimated for both groups.
\end{abstract}

Keywords: Lactation curve; crossed cows F-1 and Gir; methods; modeling and analysis.

\section{Introducción}

El Perú, así como la mayoría de países tropicales de América Latina, se caracteriza por tener grandes áreas de terreno aún no explotadas, un acelerado crecimiento demográfico y un bajo consumo per cápita de leche por habitante (70,5 kg/hab./año para el año 2013).

La producción de leche en el Perú viene demostrando un incremento sostenido durante los últimos 8 años a un nivel de 5,0 por ciento anual, pasando de 1347000 (2006) a 1821724 (2013) toneladas. En la región San Martín, se observa un crecimiento más impactante, alcanzando durante un periodo de 12 años, un incremento promedio de 58,5 por ciento anual. Este incremento en la producción de leche se vio mayormente influenciado por el sustancial crecimiento de la población de bovinos (109 199 cabezas mas), que para la zona del trópico peruano creció en un nivel de 59 por ciento desde el año 1994 hasta el 2012 (MINAGRI 2013-IV Censo Agropecuario).

\footnotetext{
${ }^{1}$ Bachiller en Ciencias Agronómicas.

${ }^{2}$ Profesor Principal, Universidad Nacional Agraria La Molina, Lima, Perú. Email: jalmeyda@lamolina.edu.pe

3 Ingeniero Zootecnista
} 
Uno de los mayores problemas de la región San Martín es la limitada disponibilidad de animales de la raza o cruces más adecuada para la realidad tropical como son el Gir Lechero y su cruce con Holstein (F-1). De estos la raza Gir tiene las características de adaptación a la zona demostrando buenos rendimientos. Actualmente la raza Gir lechero viene ejerciendo un destacado papel en el contexto de la mejora genética de la ganadería bovina de la zona tropical, por ser una raza que incorpora rusticidad, productividad y longevidad y; además porque es utilizado por los criadores como base para hacer los cruzamientos para producir animales media sangre: F-1 (50 por ciento Gir +50 por ciento de raza europea). El conocimiento de las características de la campaña productiva permitirá a los productores contar con una valiosa herramienta para hacer selección de vacas Gir lechero y Cruzadas F-1; bajo las condiciones de trópico. El estudio de la curva de lactación permitirá tomar decisiones de producción y manejo ganadero.

La producción y descripción más exacta de las curvas de lactancia en vacas de diferentes genotipos, permiten entender mejor los sistemas de producción, proporcionará información útil en el desarrollo de estrategias de selección y ayudará a optimizar la eficiencia productiva, así como permitirá modificar genéticamente las curvas de lactancia (Ramírez et al., 2004). Los mismos autores indican, que para describir la producción de leche a través de la lactancia de animales domésticos se han propuesto diversos modelos matemáticos, agregando que en ganado lechero la modelación de curvas de lactancia ha sido objeto de extensas investigaciones, afirmando que la ecuación más ampliamente utilizada fue derivada de la función Gamma incompleta que propuso Wood (1967).

Al respecto Apaza et al. (2016) determinaron que el modelo propuesto por Wood (1967) resultó ser el más adecuado en la determinación de los parámetros que definen la curva de lactancia, así como para estimar la producción de leche en vacas mestizas del altiplano (Bolivia). Afirman además que el conocimiento de la curva de lactancia permite predecir el desempeño futuro de las vacas, la persistencia de la producción y el tiempo en alcanzar el pico de producción y ajustar por día en leche de vacas que no han terminado la lactancia.

En el mismo sentido Cañas et al. (2008) al estimar curvas de lactancia en ganado Holstein y Hastíen por BON en el trópico colombiano por medio del modelo Gamma incompleto y aplicar el efecto del grupo genético y algunos factores ambientales sobre los coeficientes de la curva de lactancia encontraron diferencias significativas entre los grupos raciales para las características: producción inicial, producción total, duración de la lactancia, producción de leche en el pico, producción por día, pendiente o grado de inclinación posterior al pico y concluyeron que los coeficientes estimaos a partir del modelo Gamma incompleto están altamente influenciados por el grupo genético, por el número de partos y en menor medida por la época climática.
Aspilcueta et al. (2008) al evaluar las metodologías: función lineal, función no lineal (Gamma incompleta) y polinomios segmentados para el ajuste de la curva de lactación media en el sistema semiextensivo de vacas con composición genética con altos niveles de Brown Swiss en Tingo María, encontraron que el método polinomios segmentados muestran buen ajuste a la curva de lactación, determinando una producción total de leche para la curva media de $2123 \mathrm{Kg}$ con 272 días de lactación, una producción media de inicio de $8,0 \mathrm{~kg}$, el pico de producción se alcanzó a la $7{ }^{\mathrm{ma}}$ semana de lactación y con un promedio de 9,49 kg de leche. También demostraron que la función lineal hiperbólica muestra que el ajuste no es buena y que la función Gamma incompleta acompaña muy bien a la curva media observada. Finalmente determinaron que las funciones utilizadas, polinomios segmentados se observa que los coeficientes de determinación ajustados son semejantes y altos mostrando valores de 93,3 y 98,3 por ciento respectivamente.

Teniendo en cuenta que en el trópico peruano son escasas las investigaciones que caracterizan las curvas de lactación de bovinos se requiere conocer metodologías que permita evaluar y explicar correctamente las características de la curva de lactación de las vacas en producción, el objetivo del presente trabajo fue estudiar los métodos de regresión: lineal simple, cuadrático y no lineal de Wood, para modelar y analizar las características de la curva de lactación de vacas Gir lechero puras y cruzadas F-1 (Gir por Holstein) bajo condiciones de trópico.

\section{Materiales y métodos}

De la zona del estudio: El presente trabajo de investigación se realizó en el fundo Negociación Agrícola Ganadera San Simón ubicado en el distrito de Piscoyacu de la provincia de Huallaga, región San Martín, localizado en la parte nororiental del Perú a $1600 \mathrm{~km}$ de Lima y a 230 msnm, cuyas coordenadas son: latitud $06^{\circ} 35^{\prime}$ y longitud de $76^{\circ} 19^{\prime}$. El clima es de tipo tropical cálido-seco, con una temperatura promedio mensual de $27,05{ }^{\circ} \mathrm{C}$. La precipitación promedio anual es de $1057,5 \mathrm{~mm}$, con una humedad promedio de $80,5 \%$.

De los registros de producción: Para el modelamiento y análisis de la curva de lactación se utilizaron los registros de producción de leche de un total de 60 vacas de primera lactación seleccionadas al azar de un lote de 600 vacas, de los cuales 30 fueron vacas cruzadas F-1 (Gir lechero $\mathrm{x}$ Holstein) y 30 Gir lechero.

Del manejo y alimentación de los animales: Vacas Gir lechero: después del ordeño por la mañana (un solo ordeño por día) salen al pastoreo en potreros con Brachiaria brizanta conjuntamente con su cría. Durante el ordeño la cría es separada de la madre, es decir la vaca es ordeñada sola en la sala de ordeño donde recibe de 2 a $3 \mathrm{~kg}$ de concentrado. 
Vacas cruzadas F-1 (Gir lechero x Holstein): son criadas en forma estabulada y ordeñadas dos veces al día. Las vacas recibieron una ración alimenticia compuesta por forraje de corte picado (Pennisetum purpureun schum cv. Cameroon o pasto morado) y concentrado a razón de 2 a $3 \mathrm{~kg} / \mathrm{vaca} /$ día.

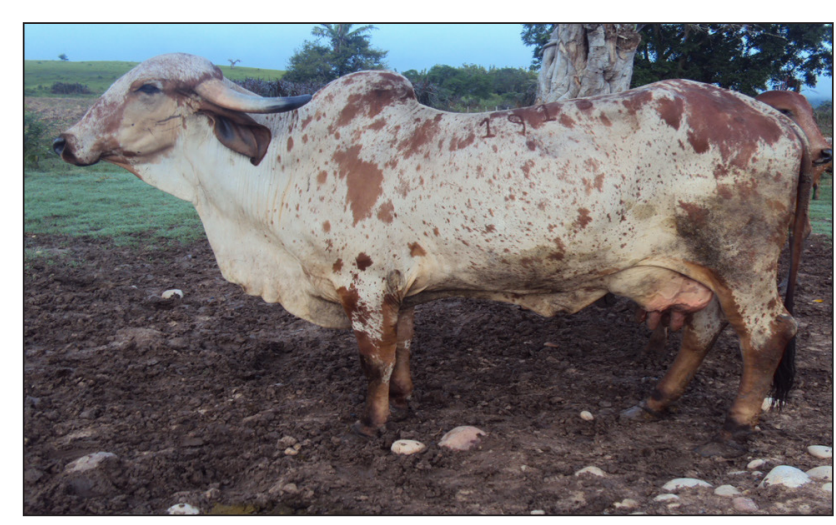

Figura 1. Vaca de raza Gir lechero

Análisis estadístico: Los métodos de regresión utilizados para modelar y analizar las características de la curva de producción de leche fueron los siguientes:

a. Método de regresión lineal simple (Madalena et al., 1979): $y(t)=a+b t$

b. Método cuadrático (Dave, 1971): $y(t)=a+b t+c t^{2}$

c. Método no lineal o función gamma incompleta de Wood (1967): $y(t)=a t^{b} e^{-c t}$

De estas tres metodologías, el método lineal incompleto o gamma incompleto, propuesto por Wood en 1967 es el más utilizado para describir curvas de lactación de: bovinos (Scherchand et al., 1995 y Vargas et al., 2000); ovinos (Pulina et al., 2001 y Afolaya et al., 2002) y cabras (Akpa et al., 2001). Este modelo matemático es una función exponencial logarítmica representada por la ecuación matemática siguiente:

$\mathrm{Yt}=\mathrm{a} \cdot \mathrm{t}^{\mathrm{b}} \cdot \mathrm{e}^{-\mathrm{ct}}$

Donde:

$\mathrm{Yt}=$ Producción de leche el día t;

$\mathrm{a}=$ Parámetro para el nivel de producción;

$\mathrm{t}=$ Día de lactancia;

$\mathrm{b}=$ Parámetro para el ritmo de aumento;

$\mathrm{e}=$ Base de logaritmo natural $(2,718) ; \mathrm{y}$,

$\mathrm{c}=$ Tasa de descenso después del pico de producción.

Este método fue propuesto por Wood (1967), donde la función potencia " $\mathrm{t}$ " " permite integrar la fase ascendente de la lactación (inicio de la producción de elche hasta el pico de producción) y el termino exponencial "-ct" la fase decreciente después del pico de producción. Por esta razón este autor interpreta los parámetros "b" y "c" como los índices de intensidad de crecimiento y decremento de la producción de leche respectivamente y como "factor de forma de la curva" a la función " $\mathrm{t}^{\mathrm{b}} \mathrm{e}^{-\mathrm{ct}}$ ", el parámetro " $\mathrm{a}$ " queda asociado al nivel de producción promedio del inicio de la lactación.

Para su solución, se utilizó el procedimiento de regresión no lineal (NLIN del Programa Statistical Analysis System, SAS V9.2, 2002) con el objetivo de calcular los parámetros "a", "b" y "c", con el fin de definir las siguientes variables:

a. Producción de leche en el pico de la curva de lactación $=\mathrm{a}(\mathrm{b} / \mathrm{c})^{\mathrm{b}} \cdot \mathrm{e}^{-\mathrm{b}}$;

b. Tiempo para alcanzar el pico de producción de leche de la campaña $=b / c ; y$,

c. Persistencia de la curva de lactación después del pico de producción $=-(b+1) \cdot \log _{n} c$

\section{Resultados y discusión}

De la curva de lactación para vacas cruzadas F-1 de primer parto: Al realizar el análisis de los métodos de regresión utilizados para evaluar las lactaciones de las vacas cruzadas F-1 (Gir lechero x Holstein), se halló resultados más confiables para el modelamiento así como para la determinación de las características de la curva de lactación cuando se utilizó el método no lineal de Wood con un coeficiente de determinación $(\mathrm{R} 2)=0,9312$, seguido del método cuadrático con un $\mathrm{R} 2=0,3919$ y por último el método lineal simple con un $\mathrm{R} 2=0,2919$, tal como se muestra en la Tabla 1.

Este resultado concuerda con lo indicado por Apaza et al. (2016) quienes manifestaron que el modelo propuesto por Wood es el más adecuado en la determinación de los parámetros que definen la curva de lactación así como para estimar la producción de leche en vacas mestizas en el Altiplano - Bolivia.

De la misma forma concuerda con lo indicado por Cañas et al (2008) quienes concluyeron que los coeficientes estimados a partir del modelo de Wood para estimar curvas de lactación en ganado Holstein y Holstein por BON en el trópico colombiano, fue el más adecuado.

De la misma forma Aspilcueta et al. (2008) encontraron al utilizar la función Gamma incompleta, acompaña muy bien a la curva media observada al ajuste de la curva de lactación en un sistema semi extensivo de vacas con composición genética con altos niveles de Brown Swiss. Los mismos autores demostraron que la utilización del método de polinomios segmentados permitirá un buen ajuste de la curva de lactación, las mismas que fueron garantizadas al obtener coeficientes de determinación con valores de 93,3 y 98,3 porciento.

Sin embargo este método propuesto por Wood difiere a lo indicado por Cerón y Corrales (2011) quienes al trabajar con datos de vacas mestizas encontraron un valor de $\mathrm{R}^{2}$ mayor con el modelo cuadrático, lo cual según sostienen, posee un mejor grado de ajuste para interpretar las características y el modelamiento de la curva de lactación de estos animales manejados bajo un sistema de doble propósito. 
Asimismo, se encontró que tanto el método no lineal de Wood, cuadrático y lineal simple fueron altamente significativos $(\mathrm{P}<0,01)$.

Tabla 1. Coeficiente de determinación $\left(\mathrm{R}^{2}\right)$ de los métodos de regresión para la curva de lactación de vacas cruzadas F-1 de primera lactación

\begin{tabular}{|c|c|}
\hline $\begin{array}{l}\text { Método } \mathrm{de}_{\text {Ecuación de regresión }} \\
\text { regresión }\end{array}$ & $\mathrm{R}^{2}$ \\
\hline $\begin{array}{l}\text { No lineal de } \\
\quad \text { Wood }\end{array}$ & 0,9312 \\
\hline 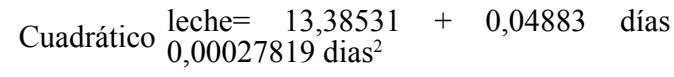 & ${ }^{-} 0,3919$ \\
\hline $\begin{array}{l}\text { Lineal } \\
\text { simple }\end{array} \quad$ leche $=17,9661-0,03495$ días & 0,2919 \\
\hline
\end{tabular}

Mediante el método de regresión no lineal de Wood, al analizar 30 lactaciones de vacas cruzadas F-1 de primer parto, se encontró que el modelo fue altamente significativo $(\mathrm{P}<0,01)$ para evaluar la curva de lactación. Asimismo, el método determinó un factor escala asociado al nivel de producción de 0,9993 , un índice de incremento de la producción de leche de 0,8376 y un índice de descenso de la producción de leche después del pico de 0,0103 (Tabla 2); los mismos que tuvieron un coeficiente de determinación $\left(\mathrm{R}^{2}\right)$ de 0,9312 valor que indica que el 93,12 por ciento de la estimación de las características de la curva de lactación es explicada por el modelo.

De la misma forma mediante el método no lineal de Wood al desarrollar la ecuación correspondiente, se determinó que a los 81 días después del parto las vacas cruzadas F-1 alcanzaron el pico de producción de leche, con un nivel máximo de 17,22 kg/día y un grado de inclinación de la curva después del pico de 8,4 por ciento; valor considerado de baja persistencia para vacas especializadas de primer parto (Tabla 3).

Estos valores encontrados por el método de Wood difiere a lo encontrado por Aspilcueta et al. (2008), que evaluaron el ajuste de la curva de lactancia en vacas de alta composición genética de Brown Swiss, donde el pico de producción fue alcanzado a la $7{ }^{\mathrm{ma}}$ semana con los niveles de producción de $9.49 \mathrm{~kg}$ de leche/día.

Tabla 2. Parámetros de la curva de lactación según el método no lineal de Wood para vacas cruzadas F-1 de primera lactación

\begin{tabular}{ll}
\hline Parámetros según método no lineal de Wood & Vacas F-1 \\
\hline a: Factor asociado al nivel de producción de leche & 0,9993 \\
b: Índice de incremento de la producción. & 0,8376 \\
c: Índice de descenso de la producción. & 0,0103 \\
\hline
\end{tabular}

En la Figura 3, se observa la modelación gráfica de la curva de lactación obtenida mediante el método cuadrático:
Producción de leche $=13,38531+0,04883$ días de lactación - 0,00027819 (días de lactación) ${ }^{2}$

Asimismo, se determinó la producción de leche por campaña de vacas cruzadas F-1 de primer parto a los 305 días por este método cuyo valor fue $3927,18 \mathrm{~kg}$ (Tabla 4).

Tabla 3. Características de la curva de lactación según el método Wood de vacas cruzadas F-1 de primera lactación

\begin{tabular}{lc}
\hline Características de curva de lactación & Vacas F - 1 \\
\hline Pico de producción (días) & 81 \\
Producción máxima de leche en el pico (kg/día) & 17,22 \\
Persistencia de la curva de lactación $(\%)$ & 8,408 \\
\hline
\end{tabular}

La modelación gráfica de la curva de lactación mediante el método no lineal de Wood se observa en la Figura 2, la cual fue obtenida mediante la siguiente fórmula:

Producción de leche $=0,9993 *$ días de lactación ${ }^{0,8376} *$ $\mathrm{e}^{-0,0103 \text { días de lactación }}$.

Finalmente, con este método de regresión no lineal de Wood, se determinó la producción de leche de la campaña de vacas cruzadas F-1 de primera lactación a los 305 días cuyo valor fue: $4031,15 \mathrm{~kg}$ (Tabla 4).

Tabla 4. Producción de leche estimada según los métodos de regresión evaluados, para vacas cruzadas F-1 de primera lactación

\begin{tabular}{lcc}
\hline \multicolumn{2}{c}{ Método de regresión Producción de leche estimada } & $\mathrm{R}^{2}$ \\
& a 305 días de lactación $(\mathrm{kg})$ & \\
\hline No lineal de Wood & $4,031.15$ & 0,9312 \\
Cuadrático & $3,927.18$ & 0,3919 \\
Lineal simple & $4,240.80$ & 0,2919 \\
\hline
\end{tabular}

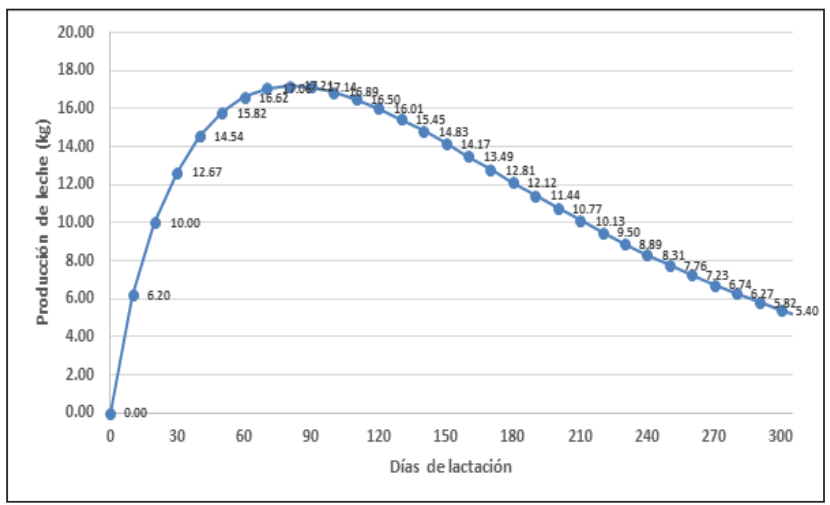

Figura 2. Modelación de la curva de lactación mediante el método no lineal de Wood para vacas cruzadas F-1 de primera lactación

En la Figura 4, se observa la modelación gráfica de la curva de lactación obtenida mediante el modelo de regresión lineal simple:

Producción de leche $=17,9661-0,03495$ días de lactación 
Asimismo, se determinó la producción de leche de vacas cruzadas F-1 (Gir lechero x Holstein) estimada a los 305 días según el método de regresión lineal simple cuyo valor fue $4240,80 \mathrm{~kg}$ (Tabla 4).

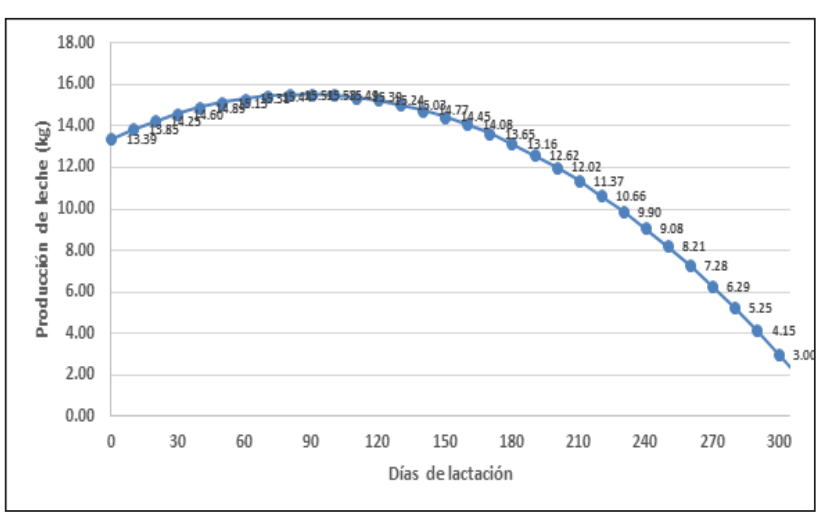

Figura 3. Modelación de la curva de lactación estimada mediante el método de regresión cuadrático para vacas cruzadas F-1 de primera lactación

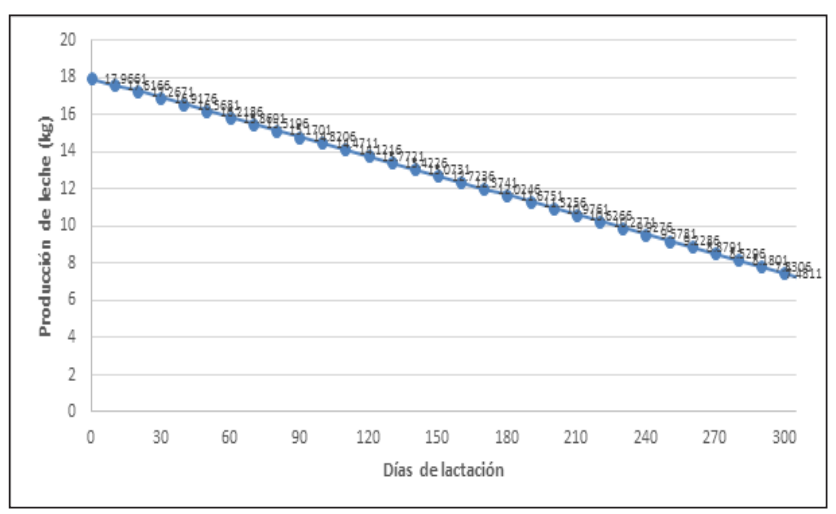

Figura 4. Modelación de la curva de lactación mediante el método de regresión lineal simple para vacas cruzadas F-1 de primera lactación

Siendo el método de regresión no lineal de Wood el que mejor explica la curva de producción de leche de vacas cruzadas F-1 de primera lactación y al realizar la estimación de la producción de leche de la campaña productiva, el valor encontrado $(4031,15 \mathrm{~kg})$ fue superior a lo reportado por De los Ríos (2011) con $3575,8 \mathrm{~kg}$ quien realizo trabajos con vacas cruzadas F-1 en Tarapoto (Perú), de la misma forma fue mejor a lo reportado por Aspilcueta et al. (2008) quienes determinaron una producción de 2 $123 \mathrm{Kg}$ de leche por campaña en vacas con latos niveles genéticos de Brown Swiss, criados en un sistema semiextensivo en Tingo María - Perú.

De la curva de lactación de vacas Gir lechero de primer parto: Al realizar el análisis de los métodos de regresión utilizados para evaluar las lactaciones de las vacas Gir lechero se encontró resultados más confiables para el modelamiento así como para la determinación de las características de la curva de lactación cuando se utilizó el método de regresión no lineal de Wood con un coeficiente de determinación $(\mathrm{R} 2)=0,9108$, seguido del método cuadrático con un $\mathrm{R} 2=0,1739$ y por último el método lineal simple con $\mathrm{R} 2=0,0846$, tal como se muestra en el Tabla 5. Asimismo, se encontró que tanto el método no lineal de Wood, cuadrático y lineal fueron altamente significativos $(\mathrm{P}<0,01)$.

Tabla 5. Coeficiente de determinación $\left(\mathrm{R}^{2}\right)$ de los métodos de regresión de la curva de lactación para vacas Gir lechero de primera lactación

\begin{tabular}{lll}
\hline $\begin{array}{l}\text { Método de } \\
\text { regresión }\end{array}$ & Ecuación de regresión & $\mathrm{R}^{2}$ \\
\hline $\begin{array}{l}\text { No lineal de } \\
\text { Wood }\end{array}$ & leche $=0,1096 *$ días $^{1,2149} * \mathrm{e}^{-0,0219 \text { dias }}$ & 0,9108 \\
Cuadrático & leche $=2,91255+0,03177$ días & $-0,1739$ \\
& 0,00025695 dias $^{2}$ & 0,0846 \\
\hline
\end{tabular}

Mediante el método de regresión no lineal de Wood al analizar 30 lactaciones de vacas Gir lechero de primer parto, se encontró que el modelo fue altamente significativo $(\mathrm{P}<0,01)$ para estimar la curva de lactación. Asimismo el método determinó un factor de escala asociado al nivel de producción de leche de 0,1096, un índice de incremento de la producción de leche de 1,2149 y un índice de descenso de la producción de leche después del pico de 0,0219 (Tabla 6) los mismos que tuvieron un coeficiente de determinación $\left(\mathrm{R}^{2}\right)$ de 0,9108 , valor que indica que el 91,08 por ciento de la estimación de las características de la curva de lactación es explicada por el método.

De la misma forma mediante el método de regresión no lineal de Wood al desarrollar la ecuación correspondiente se determinó que el tiempo para que las vacas Gir lechero alcancen el pico de producción leche fue a los 55 días después del parto, con un nivel máximo de 4,28 kg/día y un grado de inclinación de la curva de lactación después del pico de 8,4 por ciento, valor considerado de baja persistencia para vacas de primera lactación (Tabla 7).

Tabla 6. Parámetros de la curva de lactación según el método de regresión no lineal de Wood para vacas Gir lechero de primera lactación

\begin{tabular}{ll}
\hline Parámetros según método no lineal de Wood & Gir lechero \\
\hline a: Factor asociado al nivel de producción de leche & 0,1096 \\
b: Índice de incremento de la producción de leche & 1,2149 \\
c: Índice de descenso de la producción de leche & 0,0219 \\
\hline
\end{tabular}

Tabla 7. Características de la curva de lactación según el método no lineal de Wood de vacas Gir lechero de primera campaña

\begin{tabular}{lc}
\hline Características dela curva de lactación & Gir lechero \\
\hline Pico de producción (días) & 55 \\
Producción máxima de leche en el pico (kg/día) & 4,28 \\
Persistencia de la curva de lactación & 8,464 \\
\hline
\end{tabular}


Los valores del tiempo en que se alcanza al pico así como el nivel máximo de producción de leche difieren a lo encontrado en las vacas cruzadas F-1, sin embargo el nivel de grado de inclinación de la curva de lactancia fueron similares.

El modelamiento de la curva de lactación mediante el método de regresión no lineal de Wood se puede observar en la Figura 5, la cual fue obtenida mediante la siguiente fórmula:

Producción de leche $=0,1096 *$ días de lactación ${ }^{1,2149} *$ $\mathrm{e}^{-0,0219 \text { días de lactación }}$

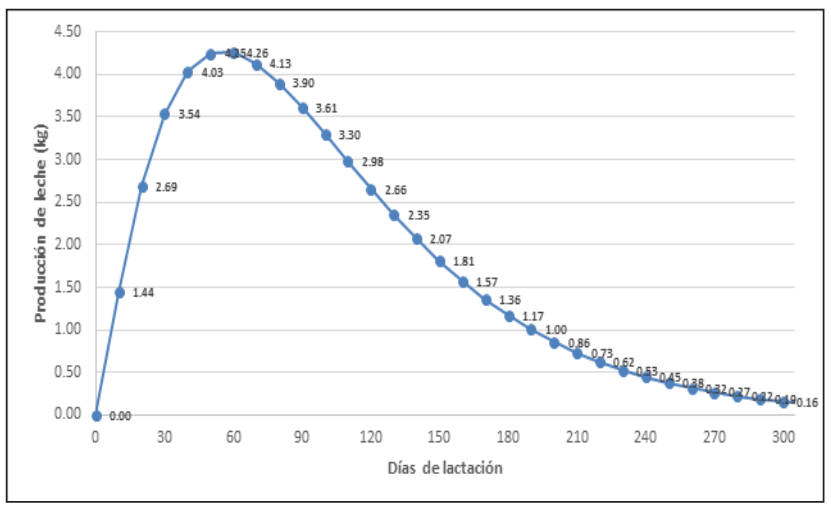

Figura 5. Modelación de la curva de lactación mediante el método no lineal de Wood para vacas Gir lechero de primera lactación

Finalmente con este método de regresión no lineal de Wood se determinó la producción de leche para vacas Gir lechero de primera lactación a los 305 días cuyo valor fue $1031,15 \mathrm{~kg}$ (Tabla 8).

En la Figura 6, se observa la modelación gráfica de la curva de lactación obtenida mediante el método cuadrático:

Producción de leche $=2,91255+0,03177$ días de lactación - 0,00025695 días de lactación ${ }^{2}$

Asimismo, se determinó la producción de leche estimada hasta los 180 días siendo el valor $1251,06 \mathrm{~kg}$ (Tabla 8), luego los valores estimados fueron negativos.

Tabla 8. Producción de leche estimada según los métodos de regresión evaluados, para vacas Gir lechero de primera lactación

\begin{tabular}{lcc}
\hline \multicolumn{1}{c}{ Método de regresión } & $\begin{array}{c}\text { Producción de leche }(\mathrm{kg}) \\
\text { estimada a 305 días de } \\
\text { lactación, a un ordeño }\end{array}$ & $\mathrm{R}^{2}$ \\
\hline No lineal de Wood & 1031,15 & 0,9108 \\
Cuadrático $(*)$ & 1251,06 & 0,1739 \\
Lineal simple & 3125,86 & 0,0846 \\
\hline (*) La estimación del método cuadrático fue hasta los 180 días, después \\
los valores estimados fueron negativos.
\end{tabular}

En la Figura 7, se observa la curva de lactación obtenida mediante el método lineal simple.

Producción de leche $=4,28805-0,01042$ días de lactación.
Asimismo, se determinó que la producción de leche para vacas Gir lechero a los 305 días fue de $3125,86 \mathrm{~kg}$ (Tabla 8).

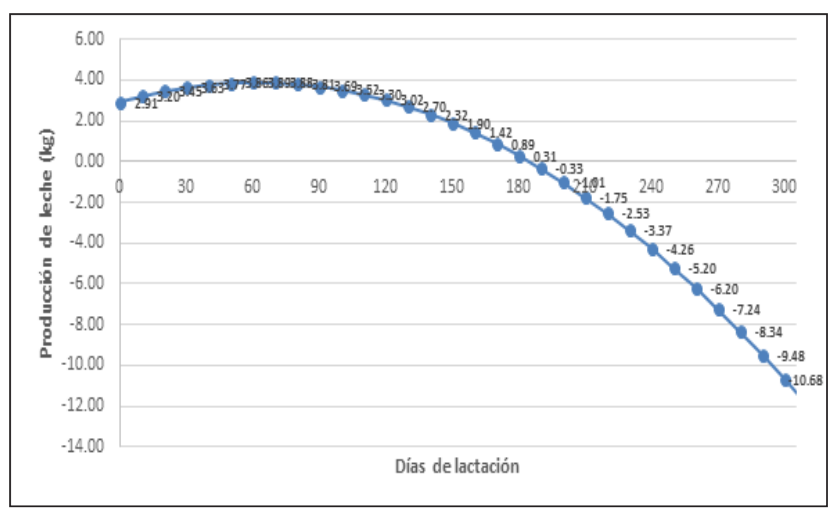

Figura 6. Modelación de la curva de lactación mediante el método de regresión cuadrático para vacas Gir lechero de primera lactación

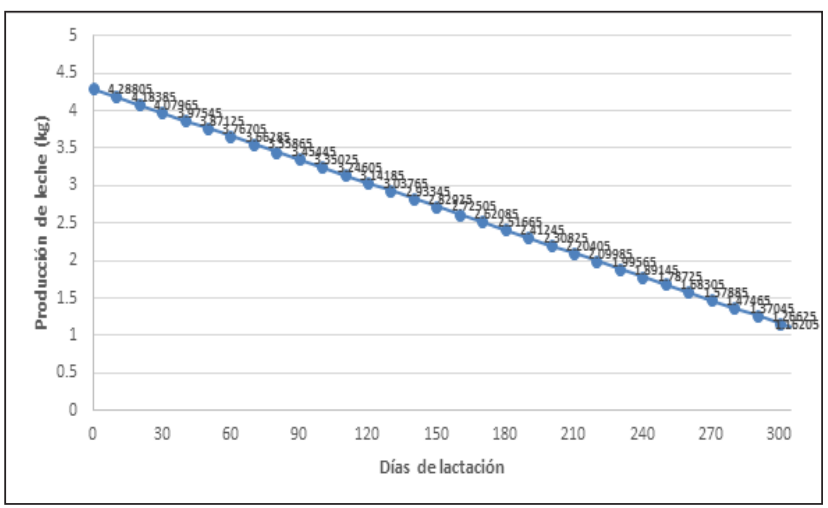

Figura 7. Modelación de la curva de lactación mediante el método de regresión lineal para vacas Gir lechero de primera lactación

Siendo el método de regresión no lineal de Wood la que mejor interpreta la curva de lactación de vacas Gir lechero de primera lactación y después de realizar la estimación de la producción de leche de la campaña productiva encontró que el valor $(1031,15 \mathrm{~kg})$ fue inferior a lo reportado por De Olivera et al. (2006) quienes indican una producción de $2818 \mathrm{~kg}$ en 260 días de duración de la campaña realizando dos ordeños por día. Es de indicar que en el caso del presente estudio las vacas fueron ordeñadas una vez por día y con presencia del ternero al pie.

Comparación entre la curva de lactación de vacas cruzadas F-1 versus Gir lechero: El método de regresión no lineal de Wood fue el que mejor explicó el modelamiento y las características de la curva de lactación (mayor coeficiente de determinación) tanto de las vacas cruzadas F-1 como de las Gir lechero. Sin embargo, los parámetros obtenidos fueron completamente diferentes para vacas cruzadas F -1 (Gir lechero por Holstein) al ser comparadas con 
las encontradas en vacas Gir lechero, ambas de primera lactación (Tabla 9).

El parámetro para nivel de producción ("a") fue mayor en vacas cruzadas F- 1 ( Gir lechero por Holstein) $(0,9993)$ comparado con el de vacas Gir lechero $(0,1096)$.

El índice para ritmo de incremento de la producción de leche antes del pico ("b") fue mayor en vacas Gir lechero con un valor de 1,12149 comparado con el de vacas cruzadas F-1 que fue de 0,8376 .

El parámetro de tasa de descenso de la producción de leche después del pico ("c") fue mayor en vacas Gir lechero con un valor de 0,0219 comparada con el de vacas cruzadas F- 1 que fue de 0,0103 .

Tabla 9. Parámetros encontrados según método de regresión no lineal de Wood para vacas cruzadas F-1 y Gir lechero

\begin{tabular}{lcc}
\hline $\begin{array}{l}\text { Parámetros según método no lineal de } \\
\text { Wood }\end{array}$ & F-1 & Gir lechero \\
\hline $\begin{array}{l}\text { a: Factor asociado al nivel de } \\
\text { producción de leche }\end{array}$ & 0,9993 & 0,1096 \\
$\begin{array}{l}\text { b: Índice de incremento de la } \\
\text { producción de leche }\end{array}$ & 0,8376 & 1,2149 \\
$\begin{array}{l}\text { c: Índice de descenso de la producción } \\
\text { de leche }\end{array}$ & 0,0103 & 0,0219 \\
\begin{tabular}{l} 
d. Coeficiente de determinación $\left(\mathrm{R}^{2}\right)$ \\
\hline
\end{tabular} & 0,9312 & 0,9108 \\
\hline
\end{tabular}

Con estos indicadores ("a", "b" y "c") se estimaron las siguientes variables productivas de las lactaciones de las vacas: días al pico de la producción, producción máxima de leche en el pico y el grado de inclinación de la curva de lactación después del pico. Los valores encontrados se observa en el Tabla 10. Al respecto, el pico de producción de leche alcanzado por las vacas cruzadas F-1 fue a los 81 días después del parto mientras que las vacas Gir lechero lo lograron a los 55 días después del parto, en cuanto al rendimiento de leche en el pico, la producción máxima alcanzada por las vacas cruzadas F-1 fue de $17,22 \mathrm{~kg} /$ día mientras que para las vacas Gir lechero lograron 4,28 $\mathrm{kg} /$ día y respecto al grado de inclinación de la curva de lactación después del pico en ambos grupos de vacas los valores encontrados fueron similares, siendo 8,408 y 8,464 por ciento para las vacas cruzadas F-1 y Gir lechero respectivamente.

Estas diferencias hace inferir que los coeficientes encontrados están altamente relacionados por el grupo genético de animales.

Tabla 10. Características de la curva de lactación para vacas cruzadas F-1 vs. Gir lechero de primera lactación

\begin{tabular}{lll}
\hline Características de la lactación & F-1 & Gir lechero \\
\hline Pico de producción (días) & 81 & 55 \\
Producción máxima leche en el pico $(\mathrm{kg})$ & 17,22 & 4,28 \\
Persistencia de la curva de lactación $(\%)$ & 8,408 & 8,464 \\
\hline
\end{tabular}

Respecto al pico de producción; para el caso de las vacas cruzadas F- 1 que lograron alcanzarlo a los 81 días después del parto el valor fue mayor a lo reportado por Sshmidt y Van Vleck (1975) quienes indican 50 a 60 días, Hafez (1986) que considera 56 a 63 días y Hutjens (2003) que refiere 40 a 60 días para vacas de razas especializadas para producción de leche manejadas en crianza intensiva.

Con referencia a las vacas Gir lechero el pico de producción se alcanzó a los 55 días después del parto siendo el valor, similar a lo indicado por los autores anteriores cuyos valores corresponden a razas especializadas para producción de leche manejadas en crianza intensiva.

En cuanto al rendimiento de leche en el pico se determinó 17,22 y 4,28 kg para vacas cruzadas F-1 y Gir lechero respectivamente. Respecto al rendimiento de las vacas cruzadas F-1 el valor encontrado fue superior a lo reportado por De los Ríos (2010) quien encontró 13,7 Kg. Es importante resaltar que las vacas Gir lechero fueron ordeñadas una vez por día.

En cuanto a la persistencia de la curva de lactación cuyos valores fueron similares tanto para las vacas cruzadas F-1 como por las Gir lechero, con 8,4 por ciento; este valor fue bastante alto comparado a lo reportado por Schmidt y Van Vleck (1975) y De los Ríos quienes indican valores de 4 a 5 y 4 para vacas de razas especializadas y cruzadas F-1 respectivamente. En este sentido es de resaltar que tanto las vacas cruzadas F-1 así como las Gir lechero de primer parto con el valor encontrado $(8,4 \%)$ muestran una curva de lactación con baja persistencia.

\section{Conclusiones}

El método de regresión no lineal de Wood fue el de mayor confianza para explicar el modelamiento y las características de la curva de lactación de vacas cruzadas F-1 y Gir lechero, de primera lactación en condiciones de trópico con coeficientes de determinación de 93,12 y 91,08 por ciento respectivamente.

Las vacas cruzadas F-1 ordeñadas dos veces por día alcanzaron una producción de $4031,15 \mathrm{~kg}$ de leche por campaña y lograron alcanzar el pico de producción de leche a los 81 días después del parto con un rendimiento de 17,22 $\mathrm{kg}$ de leche y una persistencia de $8,4 \%$, valor considerado de baja persistencia para vacas de primer parto.

Las vacas Gir lechero ordeñadas una vez por día lograron una producción de $1031,1 \mathrm{~kg}$ de leche por campaña y alcanzaron el pico de producción de leche a los 55 días después del parto con un rendimiento de 4,28 $\mathrm{kg}$ de leche y una persistencia de $8,4 \%$, valor considerado de baja persistencia para vacas de primer parto.

\section{Literatura citada}

Afolaya, R.; Abubukar, B.; Osinowo, O. and Dim, N. 2002. Lactation and function of curve parameters in Yankasa sheep. Asian - Aust. J. Anim. Sci. 15: 890 - 894.

Akpa, G.; Asiribo, E.; Oni, O. and Alawa, J. 2001. The influence of non genetic factor son the shape of 
lactation curves in Red Sokoto goats. Anim. Sci. 72: $233-239$.

Apaza Huallpa Y.; Loza Murgia, M.G.; Rojas Pardo A. y Achu Nina C. 2016. Determinación del comportamiento de la curva de lactancia y producción lechera del ganado Mestizo del Altiplano de la Provincia Omasuyos Departamento de La Paz.

Aspilcueta, R; Muñoz, M; Tonhati, H; Sesana, R; Hurtado, N. 2008. Modelación para ajuste de la curva de lactación usando función lineal, no lineal y polinomios segmentados en ganados con alto grado de sangre Brown Swiss.

Cañas, J; Restrepo, L, Ochoa, J; Echeverri, A; Cerón, M. 2008. Estimación de las curvas de lactancia en ganado Holstein y BON x Holstein en trópico alto colombiano

Ceron, M. y J. Corrales. 2011. Metodología para determinar la curva de lactación de vacas productoras de leche. Universidad de Antioquia. Colombia.

De Olivera, F; Ledic, L. Dias, M. y S. Antunes. 2006. Producao de Leite: Uma atividade economicamente viável com o Gir leiteiro.Revista Gir Leiteiro.Brasil.

De Los Rios J. 2010. Comparación de las campañas de producción de leche de vacas cruzadas F-1 (Holstein por Gir) en condiciones de trópico.

Hafez, E. 1984. Reproducción de animales de granja. México.

Hutjens, M. 2003. Guía de alimentación.Hoards Dairyman. USA.

Madalena F.E., Martinez M.L. and A. Freitas. 1979. Lactation curves of Holstein-Friesian and Holstein Friesian x Gir cows. Animal Production 29, 101-7.

MINAGRI. 2013. IV Censo Agropecuario 2012- Perú

Pulina, G.; Cappio-Borlino, A.; Macciotta, N.; Di Mauro, C. and A. Nudda. 2001. Empirical and mechanistic mathematical models of temporal evolution of milk production in ruminants. Riv. Biol. 94: 331-344.

Ramírez, R; Guadalupe, J; Núñez, R; Ruiz, A; Del Refugio, M. 2004. Comparación de ecuaciones para estimar curvas de lactancia con diferentes estrategias de muestreo en bovinos Angus, Suizo y sus cruzas.

S.A.S. INSTITUTE INC. 2002. SAS/STAT Guide for personal computers Versión 9.2. Cary, NC., USA.

Scherchand, L.; Mcnew, R.; Kelloqq, D. and Z. Johnson. 1995. Selection of a mathematical model to generate lactation curves using daily milk yields of Holstein cows. J. Dairy Sci. 78: 2507 - 2513.

Schmidt, G. y L.Van Vleck. 1975. Bases científicas de la producción lechera. Ed. Acribia. España.

Vargas, B.; Koops, W.; Herrero, M. and J. Van Arendnk. 2000. Modeling extnded lactations of dairy cows. J. Dairy Sci.. 83: 1371 - 1380.

Wood, P. D.P. (1967). Algebraic model of the lactation curve in cattle. Nature 216: 164-165.

Wood, P.D.P. (1979). A simple model of lactation curves for milk yield, food requeriment and body weight. Anim. Prod. 28:55-63 